\title{
AKT1 as the PageRank hub gene is associated with melanoma and its functional annotation is highly related to the estrogen signaling pathway that may regulate the growth of melanoma
}

\author{
JINGJING ZHAO ${ }^{1}, \mathrm{XUE} \mathrm{ZENG}^{2}$, PING SONG ${ }^{2}, \mathrm{XIAOHONG} \mathrm{WU}^{2}$ and HONGBO SHI ${ }^{1}$ \\ ${ }^{1}$ Key Laboratory of Advanced Control and Optimization for Chemical Processes of the Chinese Ministry of Education, \\ East China University of Science and Technology, Shanghai 200237; ${ }^{2}$ Department of Dermatology, \\ Guang'anmen Hospital, China Academy of Chinese Medical Sciences, Beijing 100053, P.R. China
}

Received January 15, 2016; Accepted March 1, 2016

DOI: $10.3892 / o r .2016 .5048$

\begin{abstract}
In order to detect the disease-associated genes and their gene interaction function and association with melanoma mechanisms, we identified a total of 1,310 differentially expressed genes (DEGs) from the Gene Expression Omnibus database GSE3189 with FDR $<0.01$ and $\mid \operatorname{logFCl}>2$ using the $\mathrm{R}$ package. After constructing the gene interaction network by STRING with the selected DEGs, we applied a statistical approach to identify the topological hub genes with PageRank score. Forty-four genes were identified in this network and AKT1 was selected as the most important hub gene. The AKT1 gene encodes a serine-threonine protein kinase (AKT). High expression of AKT is involved in the resistance of cell apoptosis as well as adaptive resistance to treatment in melanoma. Our results indicated that AKT1 with a higher expression in melanoma showed enriched binding sites in the negative regulation of response to external stimulus, which enables cells to adapt to changes in external stimulation for survival. Another finding was that AKT regulated the lipid metabolic process and may be involved in melanoma progression and promotion of tumor growth through gene enrichment function analysis. Two highlighted pathways were detected in our study: i) the estrogen signaling pathway modulates the immune tolerance and resistance to cell apoptosis, which contributes to the growth of melanoma and ii) the RAP1 signaling pathway which regulates focal adhesion (FA) negative feedback to cell migration and invasion in melanoma. Our studies highlighted the top differentially expressed gene AKT1 and its correlation with the estrogen signaling and RAP1 signaling pathways to alter
\end{abstract}

Correspondence to: Dr Hongbo Shi, East China University of Science and Technology, Room 202, Building 15, 130 Meilong Road, Shanghai 200237, P.R. China

E-mail: hbshi@ecust.edu.cn

Key words: melanoma, differentially expressed genes, PageRank score, AKT1, estrogen signaling pathway the proliferation and apoptosis of melanoma cells. Analysis of the enrichment functions of genes associated with melanoma will help us find the exact mechanism of melanoma and advance the full potential of newly targeted cancer therapy.

\section{Introduction}

Melanoma is a skin tumor caused by the malignant transformation of melanocytes and has an incidence rate of $4 \%$ in the US (1). Malignant melanoma is commonly characterized by rapid angiogenic growth, tumor cell apoptosis resistance, and immune tolerance.

The AKT1 gene encodes a serine-threonine protein kinase called the AKT kinase, which regulates many processes including metabolism, proliferation, cell survival, growth, and angiogenesis. High expression of AKT is involved in the resistance to cell apoptosis in melanoma (2). AKT has also been implicated in adaptive resistance to treatment. Although BRAF inhibitor (BRAFi) therapy has shown remarkable anti-melanoma responses, BRAFi therapy leads to a rebound in phosphorylated AKT levels, which result in acquired drug resistance (3).

Estrogen has been demonstrated to be involved in regulation of the immune system to allow invasion, proliferation, and migration of tumor cells such as trophoblasts (4). Increasing evidence has shown that estrogen dependency contributes to the growth of melanoma. According to results in gender-related differences, melanoma may act as a hormone-dependent tumor (5-7). The results also emphasize that melanoma is an estrogen receptor-positive tumor, whose prognosis is adversely affected by estrogen (8). In contrast, one study showed that high expression rates of estrogen receptors have no significant correlation with the prognosis of conjunctival melanoma (9). Although the effects of estrogen on the progression of melanoma are controversial, there is evidence that estrogen is the co-mediator involved in the growth of melanoma. Melanoma that is responsive to estrogens is associated with the superficial spreading melanoma subtype, a type of tumor with a much better prognosis (6). Estrogen prevents apoptosis and promotes angiogenesis, allowing melanoma to become more aggressive (10). 
In our investigations, the top hub gene named AKT1 with higher expression in melanoma showed enriched binding sites in the negative regulation of response to external stimulus, which adapts cells to changes in the external stimulation for survival. The AKT1 gene encodes a serine-threonine protein kinase (AKT). The results revealed that higher expression of AKT primarily induced the proliferation of melanoma cells. Another finding was that AKT regulated lipid metabolic process and may be involved in melanoma progression and promotion of tumor growth through gene enrichment function analysis. A high association of the estrogen signaling pathway and the RAP1 signaling pathway with melanoma was shown in this study. The estrogen signaling pathway modulates immune tolerance and resistance to cell apoptosis, which contributes to the growth of melanoma. The RAP1 signaling pathway regulates focal adhesion (FA) in a negative feedback to cell migration and invasion in melanoma.

In our study, the top hub gene AKT1 was correlated with the estrogen signaling and RAP1 signaling pathways which regulate the growth of melanoma. Screening of the differentially expressed genes (DEGs) and analysis of the enrichment functions will provide more insight into the molecular mechanisms of melanoma. Genes dependent on the kinase signaling transduction pathways are attractive targets for advanced melanoma therapy.

\section{Materials and methods}

Microarray data and preprocessing. The raw gene expression profile GSE3189 (11) was downloaded from the public database Gene Expression Omnibus (GEO, http://www.ncbi.nlm.nih. gov/geo/). In total, there were 7 normal skin and 45 melanoma samples. The corresponding platform was GPL96 (GeneChip ${ }^{\circledR}$ Human Genome U133 Set HG-U133A) Affymetrix Human Genome U133A Array which contains 22,500 human transcripts. The background correction and normalization of microarray data among microarrays was conducted by the RMA (Robust Multi-array Averaging) method (12) with the defaulted parameters in the R bioconductor package 'affy' package. Probe sets were mapped to gene symbol names using 'Annotate package'. Microarray data were filtered to extract the most variable probe set for each gene (in R software using package: genefilter).

$D E G$ screening. In order to identify DEGs, the limma $\mathrm{R}$ package (13) was used to compare the melanoma samples to the normal skin samples. The raw P-value was corrected using the Benjamin and Hochberg method (14) to circumvent the multi-test bias. The fold change value $>4$ or $<0.25$ and false discovery rate $($ FDR $)<0.01(15)$ were selected as cutoff criterion for DEGs.

Construction of the interaction network. To further analyze these DEGs, we next mapped all the DEGs to the STRING (Search Tool for the Retrieval of Interacting Genes) database (16) to construct an gene-gene interaction network. STRING integrates other databases to reveal interactions including both direct (physical) and indirect (functional) associations of the target genes. A combined score was computed by STRING, which indicates a higher confidence when more than one type of information supports a given association.

Analyzing the topological properties of the interaction network. Next we analyzed the topological properties of the interaction network, such as node degree and clustering coefficient. Node degree is the number of nodes directly connected to a node, displaying the local centrality of this node in the network. A higher node degree usually represents a stronger importance of a node for the stability of the network and a cluster coefficient usually represents how closely the adjacent nodes are connected with each other. It has already been determined that most of the biological networks are subject to scale-free network property, which means that their degree distribution follows a power law, and that the clustering coefficient distribution decreases as the node degree increases (17). These two topological properties were analyzed based on the Network Analyzer Cytoscape software (18) in the interaction network.

Hub gene identification by Google PageRank. In order to find the topological hub (important) genes, we used a weighted Google PageRank method $(19,20)$ to score all the DEGs in the interaction network. It was first applied in the Google web-search engine for identifying important web pages; it measures the importance of a node based on the sum of the rank of its backlinks (the number of nodes that link to that particular node). In addition to simply calculating the degree of each node, the PageRank score measures a gene's importance or popularity based mainly on the structure of the interaction network. Therefore, genes with lower degrees, which connect to other key genes, can also be selected as important genes.

The original PageRank is defined as:

$$
P R_{k}=\frac{1-d}{N}+d \sum_{j \in L_{k}} \frac{P R_{j}}{n_{j}}
$$

Where $L_{k}$ is the set of all the adjacent nodes connected to node $\mathrm{k}, n_{j}$ is the number of node k's adjacent nodes, $\mathrm{N}$ is the total number of the nodes in the whole network, and $d$ is the damping factor, which is usually set to 0.15 .

It is noticeable that the rank score of a network is divided evenly over the nodes to which it links; however, for the actual gene interaction network, some gene-gene associations are stronger than the others. Therefore, we modified the proposed original PageRank with weight information as Weighted PageRank (WPR):

$$
W P R_{k}=\frac{1-d}{N}+d \sum_{j \in L_{k}} \frac{P R_{j}}{n_{j}} W_{j, k}
$$

Where $\mathrm{W}_{\mathrm{j}, \mathrm{k}}$ is the combined score of the link from gene $\mathrm{j}$ to $\mathrm{k}$, which we obtained from STRING.

Gene Ontology and pathway analysis. ClueGO (21) is a Cytoscape plugin, which includes precompiled annotation files, such as GO, KEGG and BioCarta, used to analyze interrelations and enrichment of terms and functional groups in biological networks. To explore the biological function and 


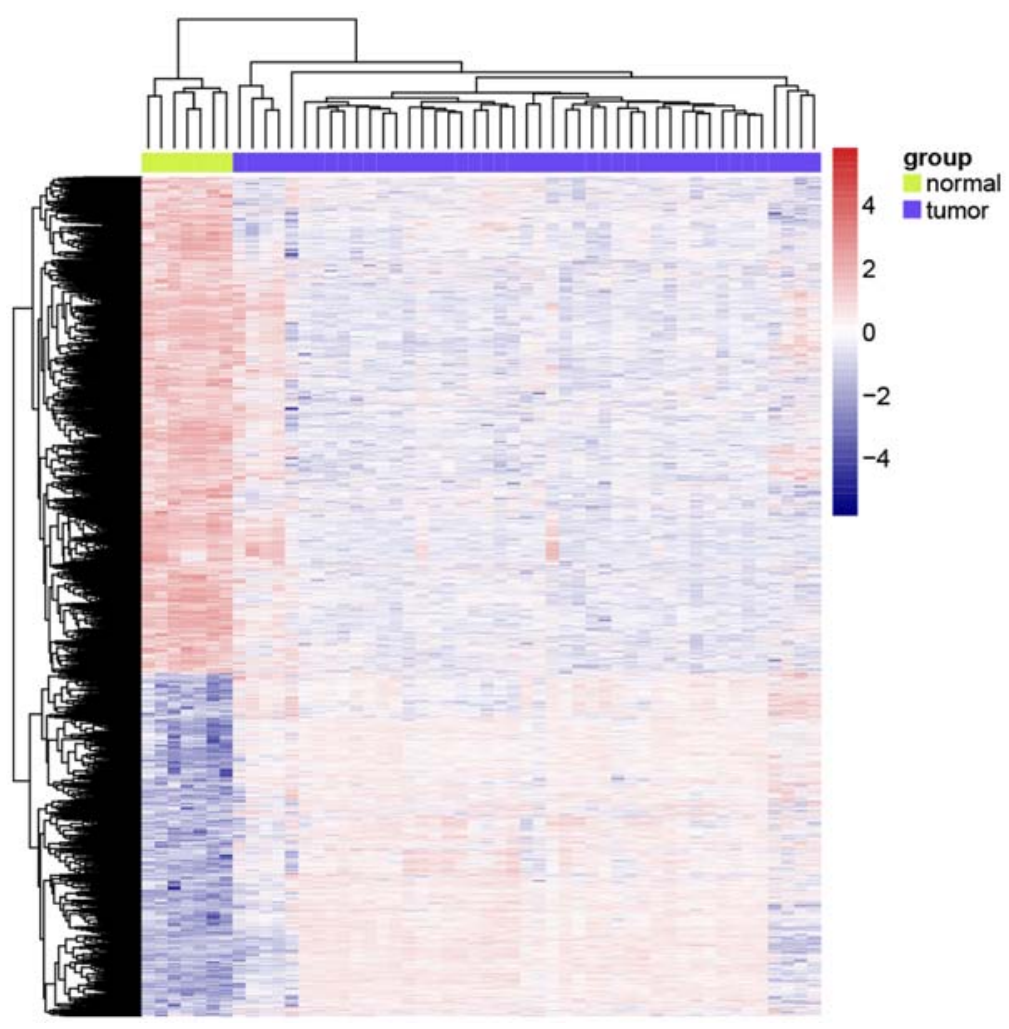

Figure 1. Heatmap of the differentially expressed genes (DEGs) between the melanoma samples and the normal skin samples.

interrelations of the top ranked important genes, we generated the different cluster of GO and pathway terms of the selected genes.

\section{Results}

Differential gene expression between melanoma and normal skin samples. The limma $\mathrm{R}$ package was used to compare gene expression profiles of melanoma and normal skin samples. At a fold change value $|\log (\mathrm{FC})|>2$ [false discovery rate (FDR) <0.01], a total of 1,310 genes were differentially expressed, including 499 upregulated genes and 811 downregulated genes; Fig. 1 shows the heatmap of all the differentially expressed genes.

Interaction network construction and topological property analysis. All the DEGs were mapped to the STRING database to construct an interaction network. STRING that is linked to other databases calculated the combined scores of the DEGs in terms of gene characteristics and spatial structures. The interaction network of the DEGs is shown in Fig. 2.

We also determined that the topological properties of the interaction network, such as node degree and clustering coefficient, are subject to scale-free network property. As shown in Fig. 3A, from the node degree distribution in the network, we obtained $y=545.19 x^{(-1.422)}$ (gray line in Fig. 3A) with the power law fitting. The $\mathrm{x}$-axis stands for the node degrees, which means the number of nodes directly connected with it, and the $y$-axis represents the number of nodes with different degrees. The property implies that the low-degree nodes belong to very dense sub-graphs and those sub-graphs are connected to each other through high degree nodes. Fig. 3B shows the topological coefficient distribution in the network, and that the clustering coefficient is able to display the aggregation degree of nodes. The nodes with a high-clustering coefficient are in the minority, and the clustering coefficient distributions are mostly located in the area between 0.1 and 0.4 .

Top PageRank gene identification and function annotation. The Google PageRank method was used to select the most important genes in the interaction network, and finally the top 44 genes with highest PageRank scores (PageRank >0.003) were selected as important genes. As shown in Table I, a high PageRank score is relatively compared to a high degree. Forty-four genes were selected as the top hub genes from 1,310 DEGs based on the PageRank score and are highlighted with red and orange color in the interaction network (Fig. 2). Unsurprisingly, AKT1 (shown as a red color gene) with upregulated expression was found to be the top gene associated with melanoma. One hundred and thirty DEGs including 26 top hub genes corresponded to AKT1.

ClueGO (Fig. 4 and Table II), revealed the enrichment and interrelation of GO terms and the pathways for these 44 genes, the cut-off of the P-value corrected with Bonferroni's method for the GO terms and the KEGG pathway are $\mathrm{P}<1.00 \mathrm{E}-4$ and $\mathrm{P}<1.00 \mathrm{E}-2$, respectively.

By using ClueGo function analysis, negative regulation of response to external stimulus was most likely associated with melanoma. As shown in Fig. 5, 9 top hub genes associated with negative regulation of response to external stimulus were detected, which included 5 downregulated genes (ANXA1, FGF2, LEP, NT5E and PPARG; colored in light blue) and 4 upregulated genes (APOE, PRKCD, SERPINE1 and TP53; colored in purple). Seven of the above 9 genes are the first 


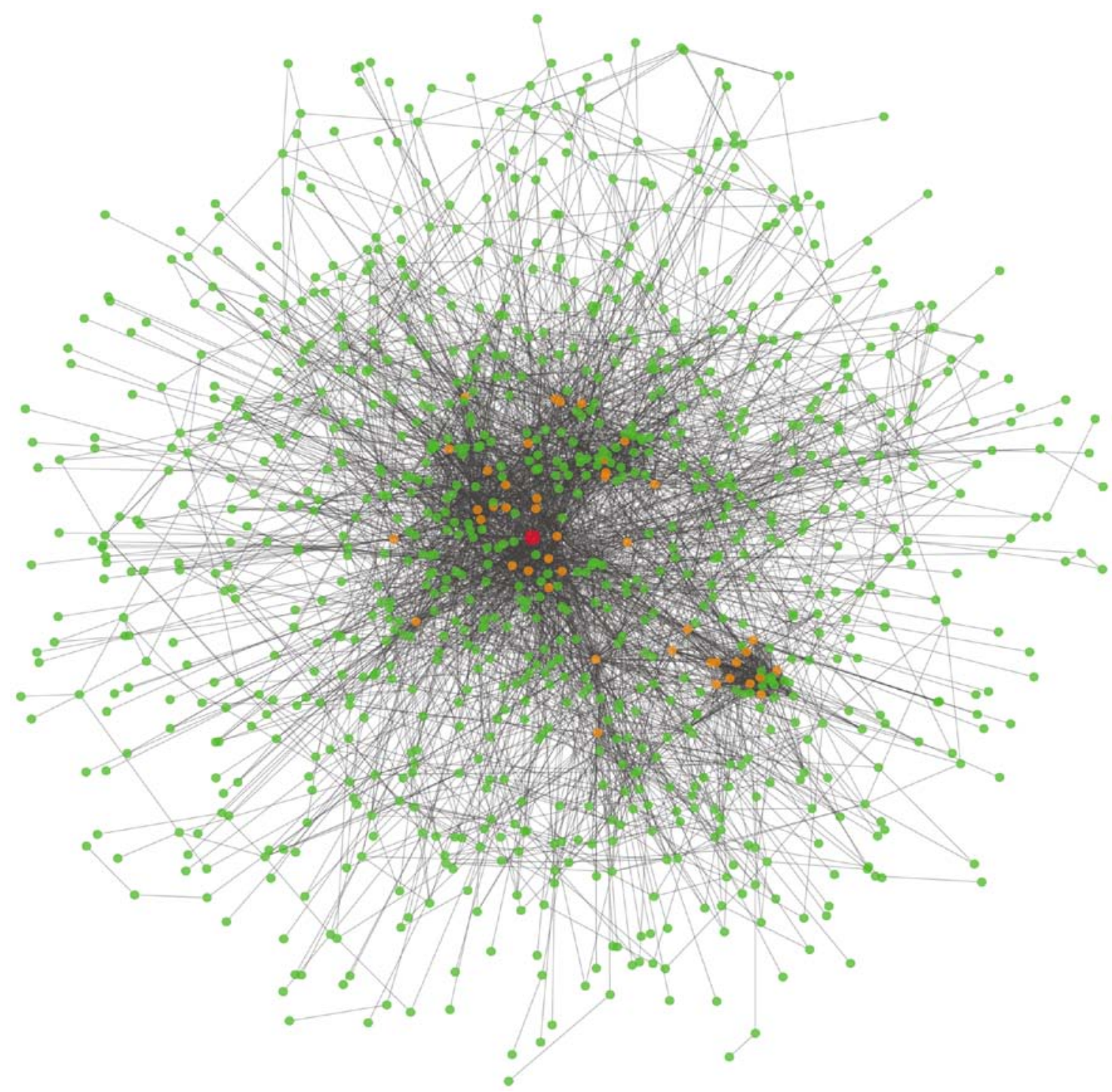

Figure 2. Interaction network of the differentially expressed genes (DEGs). The top PageRank gene, AKT1, is marked in red and the other top 43 PageRank genes are marked in orange.

neighbors of the top gene, AKT1 gene, except for ANXA1 and PRKCD.

We found that the 44 candidate genes were significantly involved in several major pathways, such as the estrogen signaling pathway, the RAP1 signaling pathway, the p53 signaling pathway, the gap junction and colorectal cancer. Eight of the 44 top hub genes are associated with the estrogen signaling pathway, including ADCY7, AKT1, EGFR, FOS, GNAI2, MMP2, PRKCD and SRC. Nine of the 44 top hub genes were associated with the RAP1 signaling pathway, including ADCY7, AKT1, EGFR, FGF2, GNAI2, IGF1R, LPAR1, RAC3 and SRC. AKT1 was shown to be involved in both pathways.

\section{Discussion}

Melanoma is a high-risk skin cancer characterized by atypical melanocyte proliferation and invasion. It is resistant to apoptosis and contributes to the growth of melanoma, which leads to metastatic melanoma, an untreatable condition.
In our study, we identified 1,310 DEGs in the gene expression profile GSE3189 (11) from the public genomics database Gene Expression Omnibus. After PageRank analysis of the interaction network, AKT1 as well as 43 other genes were selected as the top hub genes from 1,310 DEGs based on the Page-rank score (Table I).

We also analyzed the functions of the top hub genes by using ClueGo function analysis (Table II). The high significant enrichment functions of the genes associated with negative regulation of response to external stimulus were detected. Negative responses to external stimulus caused cells to adapt to changes in external stimulation for survival. Tumor cells effectuate this adaptive behavior in order to escape immune system attack by inducing immune tolerance and resisting apoptosis. We found that 9 top hub genes were highly associated with melanoma and had an effect on the negative regulation of response to external stimulus. Seven genes highly corresponded to the top one, AKT1 gene.

The AKT1 gene, which encodes a serine-threonine protein kinase named AKT kinase, was found to have 
A

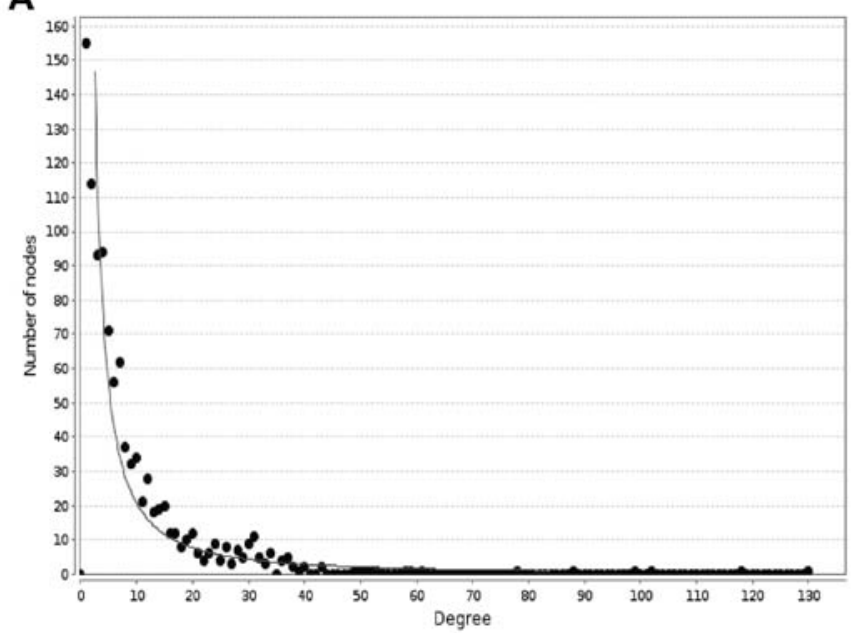

B

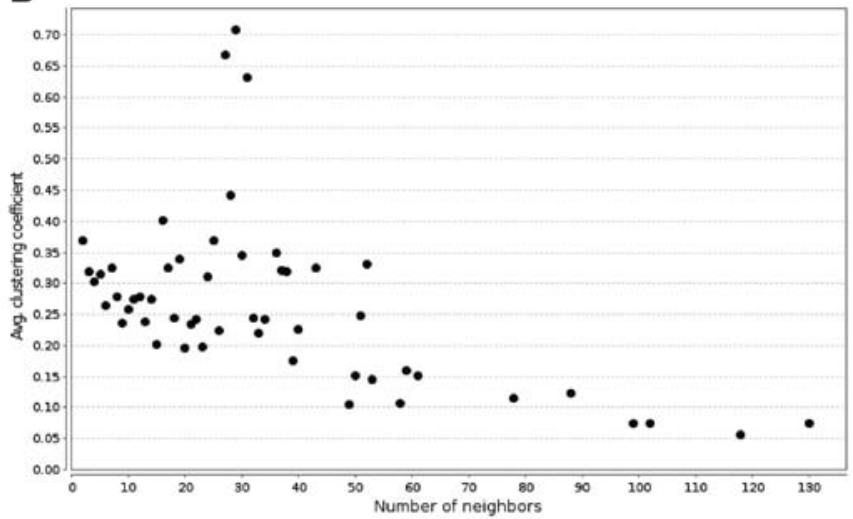

Figure 3. The topological property analysis of the network. (A) The node degree distribution. It fits in a power law model $y=545.19 \mathrm{x}^{(-1.422)}$. (B) The clustering coefficient distribution of the network.

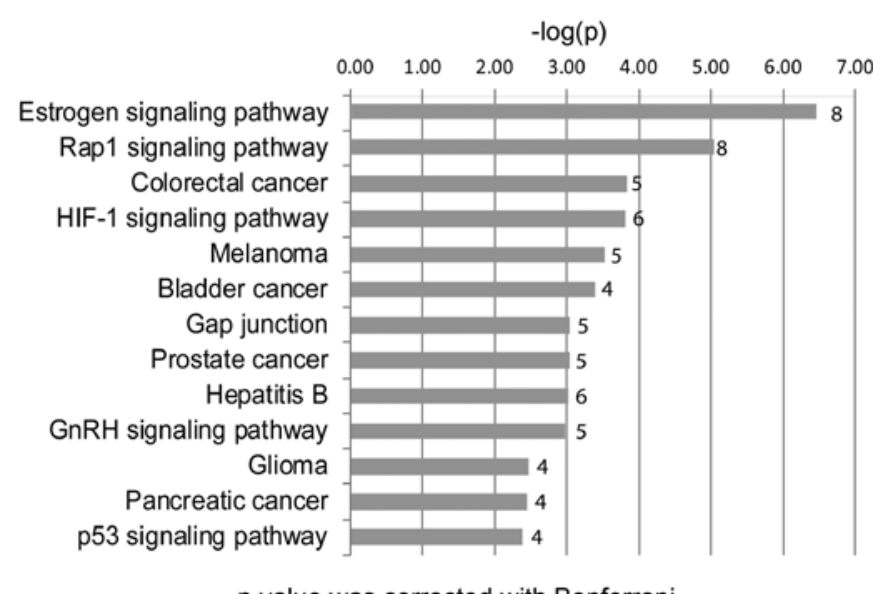

p-value was corrected with Bonferroni

Figure 4. Bar plot for the KEGG pathway enrichment analysis of the top 44 genes. The KEGG pathway enrichment P-value was corrected with Bonferroni's method. The number next to each bar represents the number of associated genes to the corresponding pathway.

higher expression in our study. The higher expression of $\mathrm{AKT}$ as well as PI3K primarily induced the proliferation of melanoma cells (22) suggesting that AKT is negatively correlated with autophagy to resist apoptosis. According
Table I. Top 44 PageRank hub genes and their topological properties.

\begin{tabular}{lcrc}
\hline Gene name & Cluster coefficient & Degree & PageRank \\
\hline AKT1 & 0.07501491 & 130 & 0.011705631 \\
TP53 & 0.05533826 & 118 & 0.010572723 \\
SRC & 0.07474277 & 102 & 0.009131527 \\
EGFR & 0.07400536 & 99 & 0.008814539 \\
GAPDH & 0.12330199 & 88 & 0.008222844 \\
FOS & 0.11488511 & 78 & 0.007051433 \\
MMP2 & 0.16014027 & 59 & 0.005436854 \\
FGF2 & 0.15136612 & 61 & 0.005407865 \\
DCN & 0.10707804 & 58 & 0.005124292 \\
CDK2 & 0.24784314 & 51 & 0.004830041 \\
PPARG & 0.14441219 & 53 & 0.00479262 \\
TYR & 0.15183673 & 50 & 0.004585681 \\
RAC3 & 0.10544218 & 49 & 0.004534422 \\
BIRC5 & 0.33107089 & 52 & 0.004446444 \\
LEP & 0.21816168 & 43 & 0.004265752 \\
NTS & 0.24615385 & 40 & 0.004015176 \\
SERPINE1 & 0.20512821 & 40 & 0.003705497 \\
FOXM1 & 0.47226174 & 38 & 0.003689789 \\
CDC45 & 0.57936508 & 36 & 0.003612235 \\
VWF & 0.1754386 & 39 & 0.003598718 \\
AURKA & 0.43078627 & 43 & 0.003555397 \\
GNAI2 & 0.26984127 & 36 & 0.003463597 \\
TYMS & 0.52552553 & 37 & 0.003439231 \\
POLR2E & 0.14962121 & 33 & 0.003425802 \\
IGF1R & 0.16642959 & 38 & 0.003385309 \\
MCM7 & 0.54654655 & 37 & 0.003349025 \\
NT5E & 0.21904762 & 36 & 0.003348752 \\
TF & 0.16666667 & 37 & 0.00331314 \\
TOP2A & 0.64171123 & 34 & 0.003299905 \\
ANXA1 & 0.32857143 & 36 & 0.003271953 \\
COL14A1 & 0.25490196 & 34 & 0.003263538 \\
NEK2 & 0.74193548 & 31 & 0.003229935 \\
MGP & 0.29032258 & 32 & 0.003214665 \\
APOE & 0.23172906 & 34 & 0.00320765 \\
PRKCD & 0.10160428 & 34 & 0.00318685 \\
CDCA8 & 0.76344086 & 31 & 0.003183032 \\
LPAR1 & 0.41935484 & 32 & 0.003169619 \\
DES & 0.11711712 & 37 & 0.003168955 \\
ADCY7 & 0.25 & 33 & 0.003111591 \\
MMP1 & 0.25225225 & 37 & 0.003101892 \\
ITGB5 & 0.14795009 & 34 & 0.003067761 \\
LMNB2 & 0.59354839 & 31 & 0.003064281 \\
CDCA3 & 0.84729064 & 29 & 0.003039415 \\
RRM2 & 0.75268817 & 31 & 0.003001865 \\
\hline & & &
\end{tabular}

to recent studies, basal autophagy is down-modulated in primary melanomas, and autophagy inhibition specifically targets the metastatic melanoma cells (2). Blocking the higher AKT activity in primary melanoma is sufficient to promote autophagy. 
Table II. GO term enrichment analysis of the top 44 genes using ClueGO.

Term P-value

corrected with

Associated genes

\begin{tabular}{lcccc} 
GO term & No. of genes & $(\%)$ & Term P-value & method \\
\hline Negative regulation of response to external stimulus & 9 & 5.142857 & $8.32676 \mathrm{E}-10$ & $4.57972 \mathrm{E}-08$ \\
Positive regulation of lipid metabolic process & 7 & 6.3636365 & $1.70611 \mathrm{E}-08$ & $8.87178 \mathrm{E}-07$ \\
Regulation of lipid biosynthetic process & 6 & 5.1282053 & $7.02603 \mathrm{E}-07$ & $3.44276 \mathrm{E}-05$ \\
Positive regulation of phospholipase C activity & 5 & 6.9444447 & $1.42718 \mathrm{E}-06$ & $6.70775 \mathrm{E}-05$ \\
Regulation of phospholipase C activity & 5 & 6.849315 & $1.52905 \mathrm{E}-06$ & $7.03362 \mathrm{E}-05$ \\
\hline
\end{tabular}

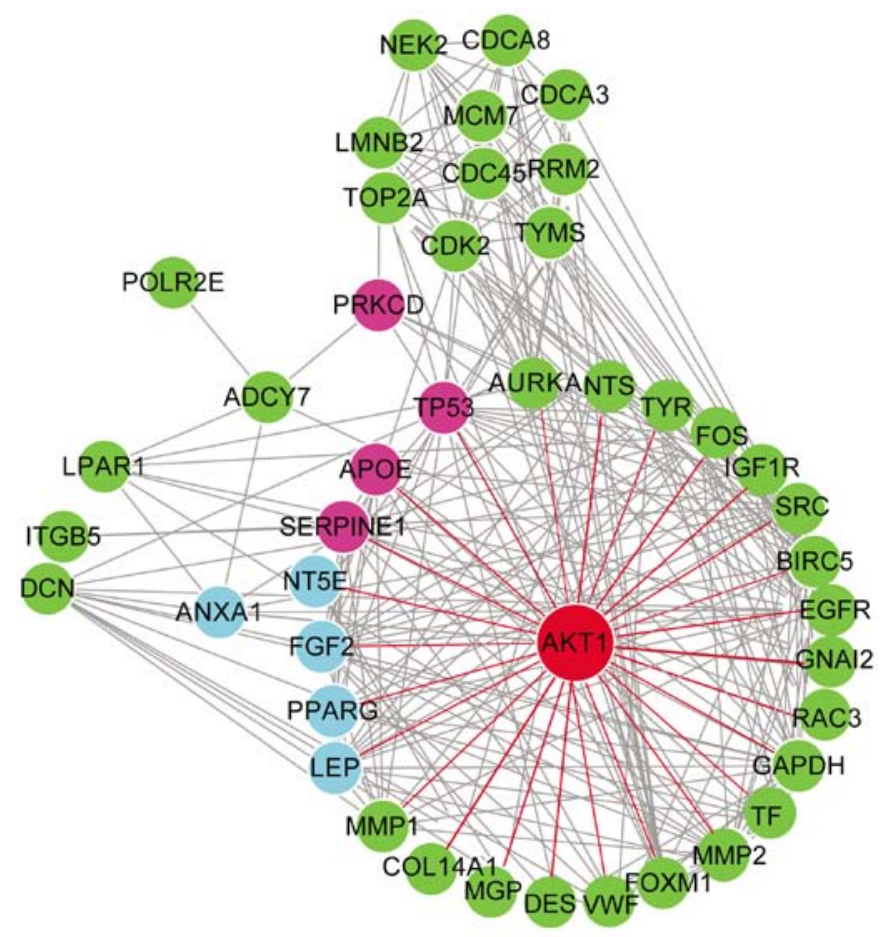

Upregulated genes associated with

negative regulation of response to external stimulus

Downregulated genes associated with

negative regulation of response to external stimulus

Figure 5. Top hub gene ATK1 and its neighbor gene network.

The RAS-RAF-MEK-MAPK and PI3K-AKT pathways play major roles in the regulation of proliferation and survival. The mutation of the BRAF gene is common in metastatic melanomas, which triggers the activation of the mitogen-activated protein kinase (MAPK) pathway-induced cell proliferation and survival (23). Depletion of BRAF as well as the BRAF gene mutation results in significantly reduced cell proliferation through inhibition of extracellular signal-regulated kinase $1 / 2$ (ERK1/2) activation and mitogen-activated protein kinase 1/2 (MEK1/2) activation (24). Therapies using BRAFi have shown a dramatic clinical efficacy in melanoma; however, the efficacy of BRAFi is short-lived due to acquired drug resistance $(25,26)$. In order to restore BRAFi sensitivity, inhibitors of the PI3K-AKT pathway as well as a mitogen-activated protein kinase inhibitor (MEKi) are required for BRAFi resistance (27). BRAFi treatment was found to lead to rebound levels of phosphatidylinositol $(3,4,5)$-trisphosphate (PIP3) and p-AKT, which participate in melanoma survival. MEK-dependent PTEN expression was found to limit PIP3 phosphate accumulation and AKT signaling (2). Thus, MAPK pathway inhibition enhances the PI3K-AKT signaling pathway and melanoma drug resistance.

An important finding was that the significantly enriched functions of melanoma top hub genes were associated with positive regulation of the lipid metabolic process and regulation of the lipid biosynthetic process.

Specifically, the AKT1 gene is involved in the aforementioned two functions suggesting that AKT regulates lipid metabolic processes and may be involved in melanoma progression and promotion of tumor growth. Recent research found that adipocytes promote melanoma cell growth by activating AKT (22), which provides evidence to confirm our hypothesis.

Through the KEGG pathway analysis, we detected that the most relevant pathway linked to melanoma is the estrogen signaling pathway and a secondary relative pathway named the RAP1 signaling pathway. The AKT1 gene is also involved in these two pathways in our study.

The most significant pathway was the estrogen signaling pathway, which is highly associated with melanoma according to our study. Augmenting the efficacy of the estrogen signaling pathway to affect the growth of melanoma requires its efficacy to focus on immune tolerance as well as apoptosis resistance. The estrogen signaling pathway modulates immune tolerance by inducing IL-10 secretion and inhibiting TNF- $\alpha$ secretion in T cells (27). Estradiol-17 (E2) at low concentrations causes the immune system to regulate immune tolerance to a non-self antigen to maintain the progression of melanoma. Estrogen can also downregulate the release of pro-inflammatory cytokines by inhibiting transcription NF- $\mathrm{kB}$ activation, which causes resistance to cell apoptosis (27).

Increasing evidence shows that the estrogen signaling pathway plays an important role in maintaining self-tolerance and modulating tolerance to non-self antigens, which contributes to melanoma proliferation. Estrogen was found to exert a proliferative effect on melanocytes and block cell cycle progression in the G1 phase, which led to the development of hyperpigmentation and to melanoma (28). Estrogen also 
prevents apoptosis. Estrogen when linked to an NOS inhibitor exerted significantly higher anti-proliferation to induce prominent apoptosis in melanoma cells (10). Angiolymphoid hyperplasia is also driven by estrogen-promoting tumor growth $(10,29)$.

The PI3K-PTEN-AKT and MAPK pathways are also involved in the estrogen signaling pathway. Tamoxifen, an anti-estrogen agent, was found to suppress phosphorylated ERK1/2 and AKT, thereby inhibiting mouse melanoma cell migration, invasion, and metastasis (30). This may explain why estrogen affects the growth of melanoma.

In our study, we also detected that the RAP1 signaling pathway was associated with melanoma, and regulates FA in a negative feedback mechanism to mediate cell migration and invasion in melanoma. RAP1-GTP-interacting adaptor molecule (RIAM) is an adapter protein involved in FA dynamics, and its depletion leads to defective melanoma cell migration and invasion through inhibition of the MEK-ERK pathway (31).

In summary, our study highlighted the top PageRank hub gene AKT1 and its correlation with the estrogen signaling and RAP1 signaling pathways to alter the proliferation and apoptosis of melanoma cells. Analysis of the enrichment functions of genes associated with melanoma show promise in elucidating the exact mechanisms of melanoma and to bring about advancements to a full potential in novel targeted cancer therapy.

\section{References}

1. Jemal A, Siegel R, Xu J and Ward E: Cancer statistics, 2010. CA Cancer J Clin 60: 277-300, 2010.

2. Maes H, Martin S, Verfaillie T and Agostinis P: Dynamic interplay between autophagic flux and Akt during melanoma progression in vitro. Exp Dermatol 23: 101-106, 2014.

3. Shi H, Hong A, Kong X, Koya RC, Song C, Moriceau G, Hugo W, Yu CC, Ng C, Chodon T, et al: A novel AKT1 mutant amplifies an adaptive melanoma response to BRAF inhibition. Cancer Discov 4: 69-79, 2014.

4. Holtan SG, Creedon DJ, Haluska P and Markovic SN: Cancer and pregnancy: parallels in growth, invasion, and immune modulation and implications for cancer therapeutic agents. Mayo Clin Proc 84: 985-1000, 2009.

5. Miller JG and Mac Neil S: Gender and cutaneous melanoma. $\mathrm{Br}$ J Dermatol 136: 657-665, 1997.

6. Enninga EA, Holtan SG, Creedon DJ, Dronca RS, Nevala WK, Ognjanovic S and Markovic SN: Immunomodulatory effects of sex hormones: requirements for pregnancy and relevance in melanoma. Mayo Clin Proc 89: 520-535, 2014.

7. Janik ME, Bełkot $\mathrm{K}$ and Przybyło M: Is oestrogen an important player in melanoma progression? Contemp Oncol (Pozn) 18: 302-306, 2014

8. Stevenson S and Thornton J: Effect of estrogens on skin aging and the potential role of SERMs. Clin Interv Aging 2: 283-297, 2007.

9. Bredow L, Stutzel L, Bohringer D, Gundlach E, Reinhard T and Auw-Haedrich C: Progesterone and estrogen receptors in conjunctival melanoma and nevi. Graefes Arch Clin Exp Ophthalmol 252: 359-365, 2014.

10. Roy S, Reddy BS, Sudhakar G, Kumar JM and Banerjee R: $17 \beta$-estradiol-linked nitro-L-arginine as simultaneous inducer of apoptosis in melanoma and tumor-angiogenic vascular endothelial cells. Mol Pharm 8: 350-359, 2011.

11. Talantov D, Mazumder A, Yu JX, Briggs T, Jiang Y, Backus J, Atkins D and Wang Y: Novel genes associated with malignant melanoma but not benign melanocytic lesions. Clin Cancer Res 11: 7234-7242, 2005 .
12. Hipfel R, Garbe C and Schittek B: RNA isolation from human skin tissues for colorimetric differential display. J Biochem Biophys Methods 37: 131-135, 1998.

13. Kerr MK. Linear models for microarray data analysis: hidden similarities and differences. J Comput Biol 10: 891-901, 2003.

14. Benjamini Y, Drai D, Elmer G, Kafkafi N and Golani I: Controlling the false discovery rate in behavior genetics research. Behav Brain Res 125: 279-284, 2001.

15. Reiner A, Yekutieli D and Benjamini Y: Identifying differentially expressed genes using false discovery rate controlling procedures. Bioinformatics 19: 368-375, 2003.

16. Franceschini A, Szklarczyk D, Frankild S, Kuhn M, Simonovic M, Roth A, Lin J, Minguez P, Bork P, von Mering C, et al: STRING v9.1: protein-protein interaction networks, with increased coverage and integration. Nucleic Acids Res 41: D808-D815, 2013.

17. Assenov Y, Ramírez F, Schelhorn SE, Lengauer T and Albrecht M: Computing topological parameters of biological networks. Bioinformatics 24: 282-284, 2008.

18. Shannon P, Markiel A, Ozier O, Baliga NS, Wang JT, Ramage D, Amin N, Schwikowski B and Ideker T: Cytoscape: a software environment for integrated models of biomolecular interaction networks. Genome Res 13: 2498-2504, 2003.

19. Page L, Brin S, Motwani R and Winograd T: The PageRank Citation Ranking: Bringing Order to the Web. Technical Report. Stanford InfoLab, 1999. http://ilpubs.stanford .edu:8090/422/.

20. Griffiths TL, Steyvers M and Firl A: Google and the mind: predicting fluency with PageRank. Psychol Sci 18: 1069-1076, 2007.

21. Bindea G, Mlecnik B, Hackl H, Charoentong P, Tosolini M, Kirilovsky A, Fridman WH, Pagès F, Trajanoski $\mathrm{Z}$ and Galon J: ClueGO: A Cytoscape plug-in to decipher functionally grouped gene ontology and pathway annotation networks. Bioinformatics 25: 1091-1093, 2009.

22. Kwan HY, Fu X, Liu B, Chao X, Chan CL, Cao H, Su T, Tse AK, Fong WF and Yu ZL: Subcutaneous adipocytes promote melanoma cell growth by activating the Akt signaling pathway: role of palmitic acid. J Biol Chem 289: 30525-30537, 2014.

23. Davies H, Bignell GR, Cox C, Stephens P, Edkins S, Clegg S, Teague J, Woffendin H, Garnett MJ, Bottomley W, et al: Mutations of the BRAF gene in human cancer. Nature 417: 949-954, 2002.

24. Calipel A, Mouriaux F, Glotin AL, Malecaze F, Faussat AM and Mascarelli F: Extracellular signal-regulated kinase-dependent proliferation is mediated through the protein kinase A/B-Raf pathway in human uveal melanoma cells. J Biol Chem 281: 9238-9250, 2006.

25. Chapman PB, Hauschild A, Robert C, Haanen JB, Ascierto P, Larkin J, Dummer R, Garbe C, Testori A, Maio M, et al: Improved survival with vemurafenib in melanoma with BRAF V600E mutation. N Engl J Med 364: 2507-2516, 2011.

26. Lo RS: Combinatorial therapies to overcome B-RAF inhibitor resistance in melanomas. Pharmacogenomics 13: 125-128, 2012.

27. Zang YC, Halder JB, Hong J, Rivera VM and Zhang JZ: Regulatory effects of estriol on $T$ cell migration and cytokine profile: inhibition of transcription factor NF-kappa B. J Neuroimmunol 124: 106-114, 2002.

28. Ribeiro MP, Silva FS, Paixão J, Santos AE and Custódio JB: The combination of the antiestrogen endoxifen with all-trans-retinoic acid has anti-proliferative and anti-migration effects on melanoma cells without inducing significant toxicity in non-neoplasic cells. Eur J Pharmacol 715: 354-362, 2013.

29. Shifren JL, Tseng JF, Zaloudek CJ, Ryan IP, Meng YG, Ferrara N, Jaffe RB and Taylor RN: Ovarian steroid regulation of vascular endothelial growth factor in the human endometrium: implications for angiogenesis during the menstrual cycle and in the pathogenesis of endometriosis. J Clin Endocrinol Metab 81: 3112-3118, 1996

30. Matsuoka H, Tsubaki M, Yamazoe Y, Ogaki M, Satou T, Itoh T, Kusunoki T and Nishida S: Tamoxifen inhibits tumor cell invasion and metastasis in mouse melanoma through suppression of PKC/MEK/ERK and PKC/PI3K/Akt pathways. Exp Cell Res 315: 2022-2032, 2009.

31. Coló GP, Hernández-Varas P, Lock J, Bartolomé RA, Arellano-Sánchez N, Strömblad S and Teixidó J: Focal adhesion disassembly is regulated by a RIAM to MEK-1 pathway. J Cell Sci 125: 5338-5352, 2012. 